\title{
AVALIAÇÃO DA GRAVIDADE DE PACIENTES INTERNADOS EM CLÍNICAS DE UM HOSPITAL ${ }^{1}$
}

\section{PARENT SEVERITY ASSESMENT IN A HOSPITAL'S CLINICAL UNITS}

\section{EVALUACIÓN DE LA GRAVEDAD DE PACIENTES INTERNADOS EN UNIDADES CLÍNICAS DE UN HOSPITAL}

\author{
TÂnia Couto Machado Chianca* \\ Helisamara Mota Guedes ${ }^{*}$ \\ Kesia MeIRIEle SouZA ${ }^{* * *}$ \\ Sara Salgado de Morais ${ }^{* * *}$ \\ Flávia Falci ErCole ${ }^{* * * *}$
}

\begin{abstract}
RESUMO
Introdução: Dados sobre a gravidade clínica dos pacientes atendidos em instituição hospitalar possibilitam direcionar a assistência de enfermagem de forma a oferecer um cuidado de maior qualidade. Objetivo: Avaliar a gravidade de pacientes internados em um hospital em relação a alta/transferência ou óbito. Método: Trata-se de uma coorte concorrente com 577 pacientes de um hospital de grande porte do Vale do Jequitinhonha, Minas Gerais, Brasil. Na admissão o paciente foi avaliado e aplicou-se o TISS-28 entre 24 e $48 \mathrm{~h}$ para a determinação da gravidade dos pacientes por meio da quantificação das intervenções de enfermagem. A análise foi procedida aplicando os testes Qui-quadrado, Kruskal-Wallis e Mann-Whitney. Resultados: Encontrou-se diferença significativa nas seguintes variáveis: idade, tempo de permanência dos pacientes e pontuação obtida pelo TISS-28 em relação ao desfecho alta/transferência e óbito. O TISS-28 mostrou uma acurácia de 77,9\% tanto na Unidade de Terapia Intensiva (UTI) como no Pronto Socorro (PS) para mensurar a gravidade clínica dos pacientes. Nas unidades de clínicas médica e cirúrgica esta foi de 65,0\%. Conclusão: O TISS-28 mostrou-se melhor instrumento para mensuração da gravidade do paciente na UTI e no PS em comparação com a daqueles internados nas clínicas.
\end{abstract}

Palavras chave: Pacientes internados, enfermagem, indice de gravidade de doença, recursos em saúde.

\begin{abstract}
Background: Collected data on the clinical severity of patients treated in a hospital provides better standards for higher nursing care quality. Objective: To assess the severity of patients admitted to a hospital in relation

\footnotetext{
${ }^{1}$ Estudo realizado com o apoio financeiro da Fundação de Amparo a Pesquisa de Minas Gerais, FAPEMIG-MG, processo de n APQ-01153-12.

* Enfermeira. Professora do Departamento de Enfermagem Básica da Escola de Enfermagem da Universidade Federal de Minas Gerais. Belo Horizonte, Brasil. Email: taniachianca@gmail.com

${ }^{* *}$ Enfermeira. Professora do Departamento de Enfermagem da Universidade Federal dos Vales do Jequitinhonha e Mucuri. Diamantina, Brasil. Email: helisamaraguedes@gmail.com

${ }_{* * *}$ Graduanda em Enfermagem pela Universidade Federal dos Vales do Jequitinhonha e Mucuri. Diamantina, Brasil. Email: kesiameiry@gmail.com

${ }^{* * * *}$ Graduanda em Enfermagem pela Universidade Federal dos Vales do Jequitinhonha e Mucuri. Diamantina, Brasil. Email: sarard1@outlook.com

${ }^{* * * * *}$ Enfermeira. Professora do Departamento de Enfermagem Básica da Escola de Enfermagem da Universidade Federal de Minas Gerais. Belo Horizonte, Brasil. Email: flavia.ercole@gmail.com
} 
to discharge/referral or death rates. Methods: A concurrent cohort of 577 patients from a large hospital in the Jequitinhonha Valley, State of Minas Gerais, Brazil. On admission the patient was first assessed and administered the TISS- 28 between 24 and $48 \mathrm{~h}$ to determine severity by quantifying nursing care interventions. The analysis was carried out applying Chi-square, Kruskal-Wallis and Mann-Whitney tests. Results: A significant difference was found in the following variables: age, length of stay in the hospital and TISS-28 score in relation to the discharge/referral and death rates. The TISS-28 showed an accuracy rate of $77.9 \%$ in measuring clinical severity of patients in both the intensive care unit (ICU) and the emergency room (ER). In medical and surgical clinics this was $65.0 \%$. Conclusion: The TISS-28 proved to be a better instrument for measuring the severity of patients in the ICU and ER compared with those hospitalized in clinics.

Key words: Inpatients, nursing, severity of illness index, health resources.

\section{RESUMEN}

Introducción: Los datos acerca de la gravedad clínica de los pacientes atendidos en una institución hospitalaria permiten orientar la asistencia de enfermería de manera de ofrecer un cuidado de mayor calidad. Objetivo: Evaluar la gravedad de pacientes internados en un hospital en relación a su alta/transferencia o muerte. Método: Se consideró una cohorte concurrente con 577 pacientes de un hospital grande del Vale do Jequitinhonha, Minas Gerais, Brasil. En la admisión el paciente era evaluado y se le aplicaba el TISS-28 entre 24 y 48 h para la determinación de la gravedad de los pacientes por medio de la cuantificación de las intervenciones de enfermería. Se procedió al análisis aplicando tests de Chi-cuadrado, Kruskal-Wallis y Mann-Whitney. Resultados: Se encontró una diferencia significativa para las siguientes variables: edad, tiempo de permanencia de los pacientes y puntuación obtenida en el TISS-28 en relación con el resultado alta/transferencia y muerte. El TISS-28 mostró una precisión de 77,9\%, tanto en la Unidad de Terapia Intensiva (UTI) como en la Sala de Emergencia (ER), para medir la gravedad clínica de los pacientes. En las unidades clínicas médica y quirúrgica la precisión fue de 65\%. Conclusión: El TISS-28 mostró ser el mejor instrumento para medir la gravedad de los pacientes en la UTI y ER en comparación con aquellos internados en las clínicas.

Palabras clave: Pacientes internados, enfermería, índice de gravedad de la enfermedad, recursos en salud.

Fecha recepción: 15/08/14 Fecha aceptación: 19/10/15

\section{INTRODUÇÃO}

As diferentes clínicas de internação de um hospital possuem características diversas de assistência multiprofissional e especialmente de enfermagem de acordo com o perfil clínico dos pacientes internados. Conhecer as características destes pacientes relativas à gravidade clínica possibilita direcionar a assistência de forma a oferecer um cuidado de maior qualidade.

Além disso, a utilização de escalas que permitem mensurar a gravidade dos pacientes possibilita a adequação da carga de trabalho da equipe obtendo um quantitativo de pessoal que assegure qualidade e adequada relação custo-benefício da assistência oferecida $(1,2)$.

Estratégias tem sido estudadas e implementadas por pesquisadores e profissionais da saúde para mensurar a severidade da doença, por meio de índices de gravidade que buscam criar escore para estimar a gravidade da doença (3).

O Therapeutic Intervention Scoring System (TISS) foi desenvolvido em 1974 por Cullem et al. (4) para mensurar a gravidade do paciente com base na quantificação de intervenções terapêuticas médicas e de enfermagem. É baseado na premissa de que independente do diagnóstico, quanto mais 
procedimentos o paciente recebe, maior é a gravidade da doença $(1,5)$.

No ano de 1996, o TISS sofreu ampla modificação, foi simplificado e deu origem ao TISS-28 (6) com o objetivo de facilitar sua aplicação prática. O sistema foi traduzido e validado para a língua portuguesa (7) e tem sido muito empregado, tanto no Brasil quanto em outros países, em Unidade de Terapia Intensiva (UTI) adulto $(2,8,9)$, com gestantes em estado crítico e em mulheres no pós-parto em UTI, em unidades de alta dependência e em salas de parto (10), UTI pediátrica $(11,12)$, pacientes cirúrgicos $(13$, 14), pacientes neurológicos (15), como parâmetro de avaliação de intervenção fisioterápica (16), com pacientes classificados pelo protocolo de Manchester $(3,17)$ em unidade de Pronto Socorro (PS).

De acordo com o somatório de pontos obtidos no TISS-28, os pacientes são classificados em quatro grupos, sendo: classe I (0 a 19 pontos) pacientes fisiologicamente estáveis e requerendo observação profilática; classe II ( 20 a 34 pontos) pacientes estáveis fisiologicamente, porém requerendo cuidados intensivos de enfermagem e monitorização contínua; classe III ( 35 a 60 pontos) pacientes graves e instáveis hemodinamicamente e classe IV (maior que 60 pontos) pacientes com indicação compulsória de internação em UTI com assistência médica e de enfermagem contínua e especializada $(1,5)$.

O TISS-28 é composto por sete grandes categorias de avaliação, sendo elas: atividades básicas, suporte ventilatório, suporte cardiovascular, suporte renal, suporte neurológico, suporte metabólico e intervenções específicas. Cada uma dessas categorias é constituída de intervenções, com pontuações que variam de um a oito. Quanto mais grave o paciente do ponto de vista clínico maior será a sua pontuação (2).

A utilização do TISS-28 traz benefícios não só para a enfermagem como para o paciente, uma vez que sua aplicação tem sido usada para o estabelecimento de dimensio- namento de pessoal. Por sua vez, um dimensionamento de pessoal compatível com a carga de trabalho do enfermeiro e profissionais capacitados podem trazer como benefício a redução da gravidade do paciente. Consequentemente, reduziria também a morbidade, mortalidade e tempo de permanência na UTI (18). Estudos tem mostrado uma relação direta entre a elevação na pontuação pelo TISS-28 e na idade dos pacientes com o desfecho óbito hospitalar, uma vez que há um aumento na intensidade dos cuidados necessários para serem dispensados a pacientes mais graves e com idades mais avançadas $(12,15,19)$.

O presente estudo surgiu da necessidade de classificar a gravidade dos pacientes internados nas clínicas de internação. A utilização de um instrumento como o TISS-28 pode permitir a previsão, provisão de recursos humanos e materiais para atender pacientes além de estabelecer a demanda de cuidados de enfermagem requeridas a partir do estabelecimento de um perfil de gravidade.

Considera-se que também possibilita uma reflexão a partir da aplicação do instrumento TISS-28 em diferentes clínicas de internação, o que ainda não foi realizado. Neste sentido, estabelecer a gravidade clínica dos pacientes atendidos na instituição é essencial para o planejamento da assistência de enfermagem.

Objetivo geral: Avaliar a gravidade de pacientes internados em um hospital em relação a alta/transferência ou óbito.

Objetivos específicos: Identificar os fatores de risco para alta/transferência ou óbito. Determinar a frequência das atividades assistenciais que compõem o escore TISS-28 em relação a alta/transferência e óbito. Classificar os pacientes segundo o grau de gravidade e, avaliar o poder preditivo do escore TISS-28 nas unidades de internação, Pronto Socorro (PS), UTI e clínica médico-cirúrgica através da Curva ROC. 


\section{MÉTODO}

Trata-se de uma coorte concorrente com 577 pacientes admitidos no Pronto Socorro (PS) da Santa Casa de Caridade de Diamantina município no Vale do Jequitinhonha, Estado de Minas Gerais, Brasil, e que foram encaminhados às três clínicas de internação (PS, UTI, clínica médica e cirúrgica). Esta instituição é referência macro e microrregional em assistência médico-hospitalar de média e alta complexidade na cidade e distritos, além de outros 35 municípios do Vale do Jequitinhonha (20).

A coleta de dados foi realizada em 4 meses consecutivos, entre maio e setembro de 2012. Ressalta-se que até no mês de agosto foram coletados dados dos pacientes internados e, após este período, interrompeu-se a entrada de casos novos. O acompanhamento dos desfechos dos pacientes incluídos do estudo foi realizado no mês de setembro. Não houve perdas e a coleta de dados aconteceu durante todos os dias da semana, incluindo finais de semana. A busca dos pacientes ocorreu diariamente com consulta em uma listagem fornecida pelo setor de internação.

Foram incluídos no estudo os pacientes maiores de 18 anos admitidos no PS, que permaneceram internados na instituição por, no mínimo, 24 h. Foram excluídos os pacientes transferidos para outro hospital e os que obtiveram alta ou faleceram antes das $24 \mathrm{~h}$ da internação.

Cálculo amostral para estimar o mínimo de pacientes a serem incluídos no estudo foi estabelecido aceitando-se um grau de confiança de $95 \%$, erro máximo permitido de $5 \%$ e uma prevalência de $50 \%$ (21). Determinou-se amostra de 370 pacientes com acréscimo de $20 \%$ para possíveis perdas, totalizando 444. A amostra final usada foi composta por 577 pacientes.

Para a coleta de dados foi utilizado um instrumento estruturado contendo dados demográficos e informações sobre a gravi- dade de cada paciente a partir da aplicação do TISS-28. Os pacientes foram classificados de I a IV, segundo a necessidade de cuidados demandada (5). Todos os pacientes foram acompanhados ao longo da internação hospitalar até o desfecho final (alta/transferência e óbito).

Considerou-se como variáveis independentes sexo, idade, tempo de permanência, clínica de internação e TISS-28. A variável dependente "desfecho" foi alta/transferência e óbito. Essas variáveis foram coletadas a partir da avaliação direta dos pacientes e consulta dos registros documentados nos prontuários dos pacientes entre 24 e $48 \mathrm{~h}$ da internação daqueles incluídos no estudo.

Não foi identificado na literatura um instrumento único que tivesse sido construído e validado para avaliar a gravidade dos pacientes nas diferentes clínicas de internação hospitalar. Optou-se por utilizar o TISS-28 por ser um instrumento traduzido e validado para o português do Brasil (7), utilizado nas clínicas $(3,17)$ e pela sua contribuição para a prática clínica.

Antes do início da coleta de dados realizou-se treinamento para os envolvidos na pesquisa baseado nas definições operacionais para aplicação do TISS- 28 conforme proposto por Padilha et al. (22), a fim de obter grau de concordância aceitável na aplicação do mesmo entre os avaliadores. O treinamento foi ministrado por especialistas que tinham experiência com instrumentos de avaliação de gravidade de pacientes em serviços de saúde. Considerou-se padrão ouro o especialista na área de urgência e emergência, com mestrado em enfermagem, pesquisa na área e experiência de mais de dois anos na utilização do TISS-28 na prática clínica.

Após o treinamento foi realizado um estudo piloto com 30 pacientes para avaliação do grau de concordância. Estes usuários passaram pela avaliação simultânea dos pesquisadores e do padrão ouro de forma independente e cega. O grau de concordância na avaliação foi calculado pelo coeficiente de 
Kappa conforme proposto por Landis e Koch (23). Este tem sido utilizado para determinar o grau de concordância entre avaliadores. A mensuração do Kappa varia de 0 a 1, valores próximos a zero indicam uma correlação pobre ou inexistente, valores próximos a um indicam correlação substancial ou perfeita. Neste estudo foram selecionados para a coleta de dados somente os avaliadores que atingiram uma concordância mínima de $0,80 \mathrm{ou}$ $80 \%$ no coeficiente de Kappa com o padrão ouro para realizar a coleta dos dados com a aplicação do TISS-28.

Os dados foram digitados e analisados no Programa Statistical Package for Social Sciences (SPSS), versão 17.0.

Foi feita análise descritiva dos dados através de frequência simples, média e mediana. Análise univariada foi procedida utilizando os testes de qui-quadrado, Kuskal Wallis e Mann-Whitney para avaliar associação entre o desfecho e as variáveis independentes (sexo, clinicas de internação, TISS-28, idade e permanência hospitalar). Foi adotado nível de significância $\mathrm{p}<0,05$ e intervalo de confiança (IC) de 95\%.

Análise multivariada foi realizada obtendo-se a Receiver Operating Characteristic Curve (ROC). Este foi o método escolhido para a avaliação da acurácia $(24,25)$ do TISS$28 \mathrm{em}$ discriminar os pacientes que tiveram alta/transferência daqueles que foram a óbito em cada clínica estudada.

Neste tem sido considerado como o melhor teste aquele em que a curva mais se aproxima do canto superior esquerdo do gráfico. $\mathrm{A}$ área abaixo da curva pode variar de $0,5 \mathrm{a}$ 1,0 . Um teste de acurácia ruim tem área sob a curva menor que 0,5 . Uma área entre $0,5 \mathrm{e}$ 0,7 indica baixa acurácia e de 0,7 a 0,9 mostra boa acurácia (24) do TISS-28 em predizer o desfecho clínico (alta/transferência e óbito).

Este estudo foi aprovado pelo Comitê de Ética em Pesquisa da UFMG sob o protocolo CAAE - 0430.0.203.000-11 e pela direção do hospital. Os indivíduos somente foram incluídos na pesquisa após a aprovação e assinatura do termo de consentimento livre e esclarecido. Com os indivíduos que se encontravam em situação de substancial diminuição em suas capacidades para o consentimento, como aqueles em estado de confusão mental e inconsciência, foi coletada a anuência com seus representantes legais.

\section{RESULTADOS}

Da coorte de 577 pacientes analisados, 58,1\% era do sexo masculino, com idade média de 58,7 anos, que permaneceu internado em média 7,3 dias no hospital e que obteve média de 15,5 pontos no TISS-28. Em relação à unidade de internação, 12,3\% foi admitido na UTI, 7,1\% no PS e 80,6\% em clínicas médicas e cirúrgicas.

Encontrou-se que a variável sexo não está estatisticamente relacionada ao desfecho $(\mathrm{p}=0,838)$, porém a idade $(\mathrm{p}<0,001)$ e o tempo de permanência $(\mathrm{p}=0,027)$ apresentaram-se como estatisticamente significativas (Tabela 1).

O tempo de permanência dos pacientes em cada setor do hospital foi de 12,32 dias $(\mathrm{IC}=9,4-15,1)$ na UTI; 6,57 dias $(\mathrm{IC}=5,9$ - 7,1) no PS e, de 6,9 dias (IC = 5,25 - 8,56) nas clínicas.

Encontrou-se associação estatisticamente significativa na pontuação do TISS-28, sendo a média entre os óbitos de 22,0 pontos (IC = 19,6 - 24,4) e a média dos que tiveram alta/ transferência de 14,7 pontos (IC = 14,0 - 15,0, $\mathrm{p}<0,001)$, o que os classifica como sendo pacientes estáveis do ponto de vista fisiológico, porém requerendo cuidados intensivos de enfermagem e monitorização contínua.

A descrição das principais procedimentos realizados nas últimas $24-48 \mathrm{~h}$ de internação esta descrita na Tabela 2.

Os pacientes foram classificados de acordo com a média do escore TISS-28, sendo que os pacientes da UTI e do PS obtiveram 26,5 pontos e 23 pontos, respectivamente, enqua- 
Tabela 1. Distribuição do sexo, idade e tempo de permanência dos pacientes que obtiveram alta, foram transferidos ou evoluíram a óbito, Diamantina, $2012(\mathrm{n}=577)$.

\begin{tabular}{|c|c|c|c|c|c|}
\hline & \multicolumn{2}{|c|}{ Alta/Transferência } & \multicolumn{2}{|r|}{ Óbito } & \multirow[t]{2}{*}{ Valor de $\mathrm{p}$} \\
\hline & $\mathrm{n}$ & IC $(95 \%)$ & $\mathrm{N}$ & IC (95\%) & \\
\hline Sexo & & & & & 0,838 \\
\hline Feminino & 211 & - & 31 & - & \\
\hline Masculino & 294 & - & 41 & - & \\
\hline Idade (média em anos) & 57,3 & {$[55,4-59,1]$} & 68,3 & {$[63,9-72,7]$} & $<0,001$ \\
\hline Tempo de permanência (média em dias) & 6,8 & {$[6,2-7,3]$} & 10,5 & {$[7,6-13,4]$} & 0,027 \\
\hline
\end{tabular}

Tabela 2. Frequência das intervenções e procedimentos que compõem o escore TISS-28 dos pacientes que tiveram alta/transferência ou óbitos, Diamantina, $2012(n=577)$.

\begin{tabular}{lccccc}
\hline Procedimentos & \multicolumn{2}{c}{ Total } & \multicolumn{2}{c}{ Alta/Transferência } & \multicolumn{2}{c}{ Óbitos } \\
& & $\mathrm{n}$ & $\%$ & $\mathrm{n}$ & $\%$ \\
\hline Monitorização padrão & 100 & 532 & 87,9 & 73 & 12,1 \\
Laboratório & 79,6 & 421 & 87,3 & 61 & 12,7 \\
Medicação múltipla & 73,5 & 387 & 87,0 & 58 & 13,0 \\
Curativo rotina & 60,6 & 312 & 85,0 & 55 & 15,0 \\
Suporte ventilatório suplementar & 41,3 & 209 & 83,6 & 41 & 16,4 \\
Tratamento para melhora da função pulmonar & 38,0 & 184 & 80,0 & 46 & 20,0 \\
Intervenções específicas fora da UTI & 34,2 & 177 & 85,5 & 30 & 14,5 \\
Diurese ativa & 32,7 & 161 & 81,3 & 37 & 18,7 \\
Medida quantitativa do débito urinário & 29,3 & 138 & 78,0 & 39 & 22,0 \\
Medicação única & 26,0 & 142 & 90,4 & 15 & 9,6 \\
Cuidados com drenos & 15,2 & 63 & 68,5 & 29 & 31,5 \\
Nutrição enteral & 7,2 & 27 & 61,4 & 17 & 38,6 \\
Ventilação mecânica & 6,4 & 17 & 43,0 & 22 & 56,4 \\
Cuidados com vias aéreas artificiais & 5,9 & 14 & 38,9 & 22 & 61,1 \\
Via venosa central & 5,2 & 16 & 50,0 & 16 & 50,0 \\
Medicação vasoativa única & 5,2 & 21 & 65,6 & 11 & 34,4 \\
Intervenção específica única na UTI & 3,3 & 13 & 65,0 & 7 & 35,0 \\
Medicação vasoativa múltipla & 2,8 & 5 & 29,4 & 12 & 70,6 \\
Intervenções específicas múltiplas na UTI & 2,1 & 3 & 23,1 & 10 & 76,9 \\
Ressuscitação cardiopulmonar & 1,9 & 5 & 41,7 & 7 & 58,3 \\
Técnicas de hemofiltração & 1,8 & 11 & 100 & 0 & 0 \\
Cateter arterial periférico & 0,9 & 0 & 0 & 6 & 100 \\
Medida de pressão intracraniana & 0,8 & 2 & 40,0 & 3 & 60,0 \\
Troca frequente de curativo & 0,8 & 4 & 80,0 & 1 & 20,0 \\
Tratamento da acidose/alcalose & 0,6 & 2 & 50,0 & 2 & 50,0 \\
Reposição de grandes perdas volêmicas & 0,3 & 1 & 50,0 & 1 & 50,0 \\
Nutrição Parenteral Total & 0 & 0 & 0 & 0 & 0 \\
Monitorização do átrio esquerdo & 0 & 0 & 0 & 0 & 0 \\
\hline
\end{tabular}


drando-se na classe II do TISS-28. Esta classe representa pacientes estáveis fisiologicamente, porém requerendo cuidados intensivos de enfermagem e monitorização contínua ( 1 , 5). Nas clínicas, a média foi de 14,5 pontos, classe I, indicando pacientes fisiologicamente estáveis e requerendo observação profilática
$(1,5)$. Foi encontrada significância estatística entre os setores de internação e o TISS-28 $(\mathrm{p}<0,001)$.

Foi realizada uma análise da aplicação do TISS-28 em outros setores do hospital uma vez que a literatura relaciona a aplicação do TISS-28 a UTI (Tabela 3).

Tabela 3. Distribuição dos dados da curva ROC por clínica de internação. Diamantina, 2012 $(\mathrm{n}=577)$.

\begin{tabular}{lcccc}
\hline Clínicas & Acurácia & Ponto de Corte & $\begin{array}{c}\text { Sensibilidade } \\
\%\end{array}$ & $\begin{array}{c}\text { Especificidade } \\
\%\end{array}$ \\
\hline UTI & 0,779 & $\geq 26,5$ & 81,8 & 73,5 \\
PS & 0,779 & $\geq 23$ & 60,0 & 96,8 \\
Clínica Médico-cirúrgica & 0,65 & $\geq 14,5$ & 65,0 & 61,2 \\
\hline
\end{tabular}

Estes valores indicam que pacientes na UTI com escore TISS $\geq 26,5$ pontos tem maior probabilidade de vir a óbito do que pacientes com TISS $\leq$ que 26,5 pontos. Desta forma, pode-se afirmar que $77,9 \%$ dos pacientes avaliados teve a morte e a sobrevida corretamente prognosticadas, considerando o ponto de corte estabelecido. No PS, pacientes com pontuação no TISS- $28 \geq 23,0$ pontos tem maior probabilidade de evoluir para óbito enquanto que para aqueles que estão internados em unidades clínicas o escore diminui para 14,5 pontos.

Para o desfecho de óbito dos pacientes internados no hospital estimou-se também um ponto de corte do escore TISS-28 utilizando a área sob a curva ROC. A acurácia avaliada pela área sob a curva ROC na UTI e no PS foi de 77,9\% e nas clínicas de 65,0\% (Gráfico 1).

Gráfico 1. Curva ROC para o escore TISS-28 na alta/transferência hospitalar ou óbito, Diamantina, $2012(\mathrm{n}=577)$.

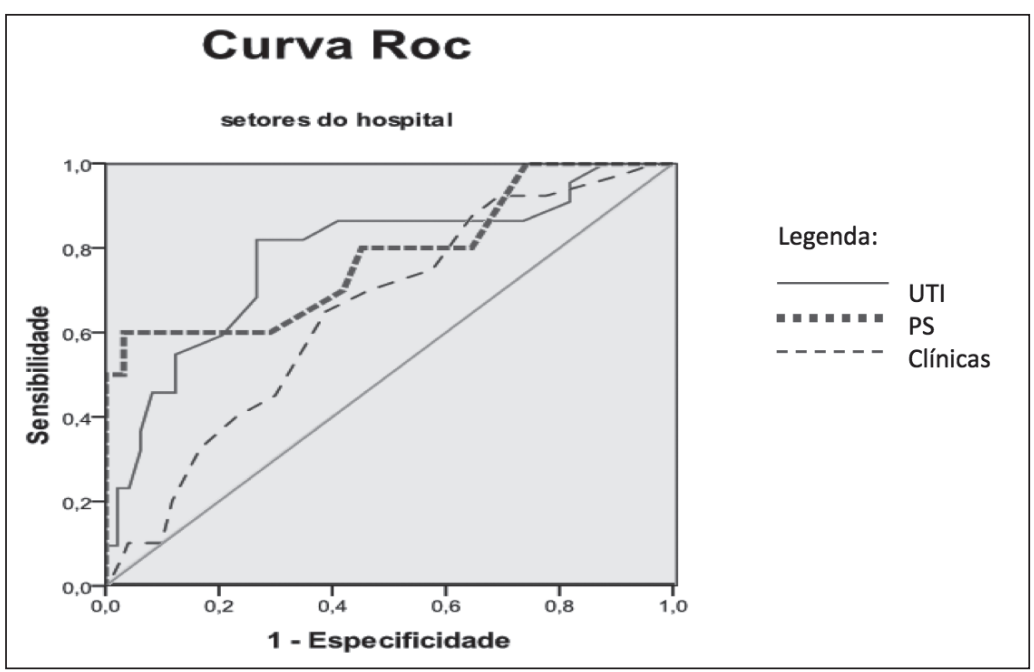




\section{DISCUSSÃO E CONCLUSÃO}

Em relação à caracterização da amostra observou-se que a média de idade dos pacientes foi de 58,1 anos, havendo um predomínio de indivíduos do sexo masculino, o que é respaldado por estudos nacionais e internacionais $(2,9,17)$.

Os dados mostraram como fator de risco para o desfecho (alta/transferência e óbito) a idade, tempo de permanência hospitalar e pontuação no TISS-28, corroborando com outros estudos internacionais $(15,19,26,27)$.

Estudo realizado para identificar fatores associados à chance de óbito hospitalar encontrou que o aumento da idade está associado com o óbito: para cada ano acrescentado na idade do paciente observou-se um aumento de $3 \%$ na chance de morte hospitalar. Encontrou-se também que cada dia de permanência hospitalar aumenta em 1\% a chance de óbito. Para pacientes na UTI a chance de óbito sobe para $4 \%$ para cada dia a mais de internação (25).

Dados sobre o desfecho óbito em ambiente hospitalar são de interesse dos profissionais de saúde, gestores, pacientes e familiares por ser este considerado um importante indicador de saúde. Em uma época em que os recursos econômicos da área hospitalar estão cada vez mais escassos, cabe aos administradores basear a decisão na alocação de recursos em ações que promovam com eficácia a prestação de cuidados. Sendo assim, o TISS28 é um bom instrumento de avaliação uma vez que quantifica as intervenções médicas e de enfermagem (28).

Estudo realizado com pacientes de UTI neurológica mostrou que a aplicação do TISS-28 no primeiro dia de internação é um bom preditor para desfechos clínicos. A identificação da gravidade no estado de saúde do paciente à admissão pode contribuir para fazer uma previsão de resultado após um ano da alta. O TISS-28 inicial pode ser usado para ajudar a decidir sobre procedimentos tera- pêuticos invasivos (15).

A média do escore TISS-28 dos pacientes internados na UTI e PS foi mais alta quando comparada a de pacientes internados nas clínicas médicas e cirúrgicas. Consequentemente, foi alta também a porcentagem de intervenções, procedimentos e monitorizações realizada com esses pacientes no período de estudo, podendo esta ser justificada pelo fato de serem internados na UTI pacientes mais instáveis do ponto de vista fisiológico e com demanda de cuidados intensivos. Por sua vez, a unidade de PS foi a segunda unidade onde os pacientes obtiveram maiores pontuações no TISS-28. Este fato pode ter ocorrido por estarem os pacientes no setor aguardando vagas nas unidades de internação.

Já era esperada uma média de pontuação elevada do TISS-28 na UTI (26,5 pontos). Estudos realizados em uma UTI de Cartagena, Venezuela e do sul do Brasil encontraram média de 28,7 (18), 26 (27) e 24,1 pontos (2), respectivamente. Esta unidade possui como características a admissão de pacientes graves que requerem tecnologias mais complexas e devido à condição patológica, possuem maior probabilidade de morte durante o período de internação (19). Os estudos acima classificam os pacientes na classe II que corresponde àqueles fisiologicamente estáveis, porém que requerem cuidados intensivos de enfermagem e monitorização.

Observa-se alto número de intervenções, monitorizações e procedimentos realizados nos pacientes internados nas clínicas médicas e cirúrgicas, sendo mais alta a taxa de realização de procedimentos nos pacientes que evoluíram a óbito em comparação com os que obtiveram alta ou foram transferidos. Quanto maior o número de intervenções e procedimentos exigidos no cuidado do paciente, mais grave será seu estado clínico, ou seja, maior será a necessidade de cuidados realizados pela equipe de enfermagem.

Estudo realizado em UTI no Brasil mostrou uma acurácia de $99,1 \%$ do TISS-28 na avaliação de pacientes que evoluíram a óbito, 
sensibilidade de $95 \%$, especificidade de $98 \%$ (1). Em uma UTI da Venezuela encontrouse um TISS $>20$ pontos que demonstra uma alta sensibilidade e baixa especificidade para predizer mortalidade. Os autores indicaram a necessidade de utilização de outras escalas para prever a evolução do paciente na internação em uma UTI (27). No presente estudo, esperava-se uma acurácia menor para as clínicas médicas e cirúrgicas tendo em vista que as demais unidades do hospital internam pacientes classificados com gravidade clínica menor.

A UTI apresentou uma acurácia igual à do PS $(0,779)$, porem a sensibilidade do TISS- 28 foi maior $(81,8 \%)$, ou seja, foi capaz de detectar o risco de morrer dos pacientes. Portanto, pacientes mais graves apresentam pontuação no TISS-28 maior e um maior risco de óbito. No PS a especificidade do TISS-28 foi maior $(96,8 \%)$ comparada com a da UTI $(73,5 \%)$. No emprego de instrumentos de avaliação em unidades de um hospital, espera-se que ele seja o mais sensível possível e o mais específico também. No caso do TISS-28 observou-se que, quando aplicado a pacientes em UTI, possui alta sensibilidade e especificidade, o que reforça sua aplicabilidade.

Por outro lado, nas clínicas médicas e cirúrgicas encontrou-se pacientes menos graves e, por isto, a acurácia do TISS-28 para predizer o óbito foi menor $(0,65)$. Encontrou-se na literatura instrumentos que se restringem a avaliação de necessidades individualizadas de cuidado de enfermagem $(29,30)$. Porém, mediante a baixa acurácia do TISS-28 (65\%) na aplicação em pacientes internados em clínicas diversas, sugere-se que outros instrumentos possam ser testados para mensurar a gravidade ou demandas de cuidados de enfermagem de pacientes em clínicas médicas e cirúrgicas.

A utilização de sistemas de classificação é um ponto de partida para planejar a carga de trabalho da enfermagem, quantificar e qualificar cuidados e fazer a provisão de recursos para um mesmo grupo de pacientes, além de alocar membros da equipe de enfermagem de acordo com necessidades reais e específicas de cada setor. A utilização de instrumentos para a classificação é útil para a gestão dos recursos e possibilita contribuir para a redução de complicações, sequelas, mortalidade e melhor qualidade de vida (18).

Os resultados do estudo mostraram que o aumento da idade, maior tempo de permanência hospitalar e maior pontuação no TISS-28 são fatores de risco para o desfecho óbito. Os pacientes que foram a óbito apresentaram um maior número de intervenções terapêuticas quando comparados com os que obtiveram alta ou foram transferidos.

A acurácia do TISS-28 foi igual na UTI e no PS, porem a sua sensibilidade na UTI é maior. Desta forma, o estudo reforça a indicação da aplicação do TISS-28 na UTI. Os resultados apontaram para uma acurácia baixa do TISS-28 nas clínicas de internação, não sendo recomendada sua utilização naquelas unidades.

Sugere-se o desenvolvimento, teste e validação de instrumentos que aplicáveis ao perfil de pacientes das clínicas médicas e cirúrgicas, com itens no instrumento que considere a utilização de tecnológicas leves, a permanência dos familiares nas $24 \mathrm{~h}$ do dia, ações de promoção da saúde, dentre outros. Porem, vale ressaltar a importância do TISS28 para a mensuração da carga de trabalho de enfermagem e que pode funcionar como facilitador na prática clínica do enfermeiro.

\section{REFERÊNCIAS}

1. Elias ACGP, Tiemi M, Cardoso LTQ, Grion, CMC. Application of the therapeutic intervention scoring system (TISS 28) at an intensive care unit to evaluate the severity of the patient. Rev Lat Am Enfermagem. 2006; 14(3): 324-29.

2. Perão OF, Bub MBC, Rodríguez AH, Zandonadi GC. Gravidade de pacientes e car- 
ga de trabalho de enfermagem em unidade de terapia intensiva. Cogitare Enferm. 2014; 19(2): 261-8.

3. Guedes HM, Martins JCA, Chianca TCM. Valor de predição do Sistema de Triagem de Manchester: avaliação dos desfechos clínicos de pacientes. Rev Bras Enferm. 2015; 68(1): 45-51.

4. Cullem DJ, Civetta JM, Briggs BA, Ferrara LC. Therapeutic intervention scoring system: a method for quantitative comparison of patient care. Crit Care Med. 1974; 2(2): 57-60.

5. Santos DS, Chianca TCM, Alvarenga AW. A systematic review study of therapeutic intervention. Rev enferm UFPE on line. 2010; 4(2):858-64.

6. Miranda DR, Rijk AP, Schaufeh W. Simplified therapeutic intervention scoring system: the TISS 28 iten-results from a multicenter study. Crit Care Med. 1996; 24(1): 64-73.

7. Nunes B. Tradução para o português e validação de um instrumento de medida de gravidade em UTI: Therapeutic Intervention Scoring System - 28 (TISS - 28). [Dissertação Mestrado em Enfermagem]. [São Paulo, São Paulo]: Escola de Enfermagem, Universidade de São Paulo, São Paulo, 2000. $91 \mathrm{f}$.

8. Klimašauskas A, Sereikè I, Klimašauskienè A, Kèkštas G, Ivaškevičius J. The Impact of Medical Conditions on the Quality of Life of Survivors at Discharge From Intensive Care Unit. Medicina (Kaunas) 2011; 47(5): 270-7.

9. Wysokinski M, Ksykiewicz-Dorota A, Fidecki W. Scope of Nursing Care in Polish Intensive Care Units. Biomed Res Int. 2013: 1-9.

10. Pollock WE, Harley NS, Nelson SM. Maternal severity of illness across levels of care: a prospective, cross-sectional study. Aust Crit Care. 2011; 24(4): 218-28.

11. Canabarro ST, Velozo KD, Eidt OR, Piva JP, Garcia PC. Validação Concorrente de Escores de Enfermagem (NEMS e TISS-
28) em terapia intensiva pediátrica. Acta Paul Enferm. 2013; 26(2): 123-9.

12. Campagner AOMP, Garcia CR, Piva JP. Aplicação de escores para estimar carga de trabalho de enfermagem em unidade de terapia intensiva pediátrica. Rev Bras Ter Intensiva. 2014; 26(1): 36-43.

13. Guimarães RCM, Rabelo ER, Moraes MA, Azzolin K. Severity of postoperative cardiac surgery Patients: na Evolution Analysis According to TISS-28. Rev Lat Am Enfermagem. 2010; 18(1): 61-66.

14. Krenzien F, Matia I, Wiltberger G, Hau HM, Schmelzle M, Jonas S, Kaisers UX, Fellmer PT. Early prediction of survival after open surgical repair of ruptured abdominal aortic aneurysms. BMC Surg. 2014; 18; 14: 92.

15. Kiphuth IC, Schellinger PD, Köhrmann M, Bardutzky J, Lücking $\mathrm{H}$, Kloska $\mathrm{S}$, Schwab S, Huttner HB. Predictors for good functional outcome after neurocritical care. Critical Care 2010; 14(4): R136.

16. Hanekom SD, Louw Q, Coetzee A. The way in which a physiotherapy service is structured can improve patient outcome from a surgical intensive care: a controlled clinical Trial. Critical Care 2012; 16(6): R230.

17. Pinto Júnior D, Salgado PO, Chianca TCM. Predictive validity of the Manchester Triage System: evaluation of outcomes of patients admitted to an emergency department. Rev Lat Am. Enfermagem. 2012; 20(6): 1041-47.

18. Romero-Massa E, Lorduy-Bolívar JP, Pájaro-Melgar C, Pérez-Duque CA. Relación entre la carga laboral de enfermería y la gravedad del paciente en unidades de cuidado intensivo de adultos. Aquichan. 2011; 11(2): 173-186.

19. Adamsk J, Goraj R, Onichimowski D, Gawlikowska E, Weigl W. The differences between two selected intensive care units located in central and northern Europe-preliminary observation. Anaesthesiol Intensive Ther. 2015; 47(2): 117-124. 
20. Santa Casa de Caridade de Diamantina [Internet]. Minas Gerais; 2015 [citado 20 outubro 2015]. Disponível em http:// santacasadecaridadedediamantina.com. br/index.php/fatos-historicos

21. Barbeta PA, Reis MM, Bornia AC. Estatística: para cursos de engenharia e informática. São Paulo: Atlas; 2010.

22. Padilha KG, Souza RM, Miyadahira AM, Cruz DA, Vattimo MF, Kimura $M$ et al. Therapeutic intervention scoring system-28 (TISS-28): diretrizes para aplicação. Rev Esc Enferm USP 2005; 39(2): 229-33.

23. Landis JR, Koch GG. The measurement of observer agreement for categorical data. Biometrics. 1977; 33: 159-74.

24. Hanley JA, Mcneil BJ. The meaning and use of the area under a receiver operating characteristic (ROC) curve. Radiology. 1982; 50(2): 3-6.

25. Nunes AA; Martinez EZ; Ana LW; PazinFilho A; Coelho EB; Mello LM. Testes diagnósticos no contexto da avaliação de tecnologias em saúde: abordagens, métodos e interpretação. Medicina (Ribeirão
Preto) 2015; 48(1): 8-18.

26. Junqueira RMP, Duarte EC. Fatores associados à chance para a mortalidade hospitalar no Distrito Federal. Epidemiol. Serv. Saúde. 2013; 22(1): 29-39.

27. Chacón-Lozsán FJ, Delgado C, Castillo Y, Pacheco PA, Narvaez MCS. Score Therapeutic Intervention Scoring System de ingreso a la unidad de cuidados intensivos como predictor de mortalidad intra-hospitalaria. Acta Colombiana de Cuidado Intensivo. 2015.

28. Capuzzo M, Ranzani OT. How objective is the observed mortality following critical care? Intensive Care Med 2013; 39: 2047-2049.

29. Perroca MG, Gaidzinski. Instrumento de classificação de pacientes de Perroca: teste de confiabilidade pela concordância entre avaliadores - correlação. Rev Esc Enferm USP. 2002; 36(3): 245-52.

30. Silva LC, Nogueira LS, Settervall CHC, Sousa RMC, Padilha GP. Desempenho de índices de gravidade para estimar risco de morte em Unidades de Terapia Intensiva. Rev Esc Enferm USP. 2012; 46(4): 846-50. 